\title{
Effect of the cosmological constant on the bending of light and the cosmological lens equation
}

\author{
Hideyoshi ARAKIDA* \\ Graduate School of Education, Iwate University, Morioka, Iwate 020-8550,Japan \\ Masumi KASAI \\ Graduate School of Science and Technology, Hirosaki University, Hirosaki, Aomori 036-8561 Japan
}

(Dated: July 23, 2018)

\begin{abstract}
We revisit the effect of cosmological constant $\Lambda$ on the light deflection and its role in the cosmological lens equation. First, we re-examine the motion of photon in the Schwarzschild spacetime, and explicitly describe the trajectory of photon and deflection angle $\alpha$ up to the second-order in $G$. Then the discussion is extended to the contribution of the cosmological constant $\Lambda$ in the Schwarzschild-de Sitter or Kottler spacetime. Contrary to the previous arguments, we emphasize the following points: (a) the cosmological constant $\Lambda$ does appear in the orbital equation of light, (b) nevertheless the bending angle of light $\alpha$ does not change its form even if $\Lambda \neq 0$ since the contribution of $\Lambda$ is thoroughly absorbed into the definition of the impact parameter, and (c) the effect of $\Lambda$ is completely involved in the angular diameter distance $D_{A}$.
\end{abstract}

PACS numbers: 95.30.Sf, 98.62.Sb, 98.80.Es

\section{INTRODUCTION}

Nowadays, it is widely regarded that the cosmological constant $\Lambda$ or more generally dark energy is the most responsible candidate which explains accelerating expansion of Universe. Nonetheless the details of cosmological constant $\Lambda$ or dark energy are still far from clear, then it is preferable and worthy to clarify the validity of this hypothesis by means of not only cosmological observations but also another astronomical/astrophysical ones.

Among such attempts, it would be the most natural idea to investigate the role of cosmological constant $\Lambda$ in the classical tests of general relativity, e.g. the perihelion advance of planetary orbit and the bending of light path. So far, it was shown that the cosmological constant $\Lambda$ causes the perihelion shift of planets at least in principle, even though its contribution is too small to detect in the current measurement technique (see [1 3] and the references therein).

While it has been believed for a long time that $\Lambda$ does not contribute to the light deflection because there is no $\Lambda$ in the second-order ordinary differential equation (ODE) of photon. However recently, Rindler and Ishak [4] pointed out that $\Lambda$ does affect the bending angle by using the Schwarzschild-de Sitter or Kottler metric and the invariant formula of cosine. Subsequently many authors argued its appearance in diverse ways and generality assisted the fact that there appears $\Lambda$ in the deflection angle $\alpha$, see [5] for review and the references therein and also 6 12]. However, it seems that the conclusion has not converged yet; for instance whether the leading order effect of $\Lambda$ is coupled with the mass of central body

\footnotetext{
*E-mail arakida@iwate-u.ac.jp

${ }^{\dagger}$ E-mail kasai@phys.hirosaki-u.ac.jp
}

$M$ or not and so on. In order to clear up the confusion, we will revisit the effect of the cosmological constant on the light deflection and its role in the cosmological lens equation.

\section{PHOTON TRAJECTORY IN SCHWARZSCHILD SPACETIME}

Before discussing the influence of $\Lambda$ on bending angle $\alpha$, we shall begin with re-considering the solution of photon trajectory in the Schwarzschild spacetime. From the Schwarzschild metric in the Schwarzschild coordinates,

$$
\begin{aligned}
d s^{2}= & -\left(1-\frac{r_{g}}{r}\right) c^{2} d t^{2}+\left(1-\frac{r_{g}}{r}\right)^{-1} d r^{2} \\
& +r^{2}\left(d \theta^{2}+\sin ^{2} \theta d \phi^{2}\right), \quad r_{g}=\frac{2 G M}{c^{2}}
\end{aligned}
$$

and the condition for null geodesic $d s^{2}=0$, we have the geodesic equation for equatorial plane $(\theta=\pi / 2)$,

$$
\left(\frac{d u}{d \phi}\right)^{2}=\frac{1}{b^{2}}-u^{2}+r_{g} u^{3}, \quad u \equiv \frac{1}{r}, \quad \frac{1}{b^{2}} \equiv \frac{E^{2}}{c^{2} L^{2}},
$$

in which the two constants of motion, $E$ and $L$ are total energy and angular momentum, respectively. Alternatively, Eq. (2) can be expressed in the form of secondorder ODE as,

$$
\frac{d^{2} u}{d \phi^{2}}=-u+\frac{3}{2} r_{g} u^{2}
$$

nevertheless, hereinafter we use Eq. (2) instead of Eq. (3).

In order to obtain the photon trajectory up to the second-order in $G$, let us put the solution of Eq. (2) $u$ as,

$$
u=\frac{1}{b}\left(\sin \phi+r_{g} u_{1}+r_{g}^{2} u_{2}\right)
$$


where $u_{1}$ and $u_{2}$ are, respectively, first $\mathcal{O}(G)$ and second $\mathcal{O}\left(G^{2}\right)$ order correction to zeroth-order solution $u_{0}=$ $\sin \phi / b$ (straight line). Hence $u_{1}$ and $u_{2}$ satisfy the following differential equations,

$$
\begin{aligned}
\frac{d u_{1}}{d \phi}= & -\frac{\sin \phi}{\cos \phi} u_{1}+\frac{1}{2 b} \frac{\sin ^{3} \phi}{\cos \phi} \\
\frac{d u_{2}}{d \phi}=- & -\frac{\sin \phi}{\cos \phi} u_{2} \\
& \quad-\frac{1}{2 \cos \phi}\left[\left(\frac{d u_{1}}{d \phi}\right)^{2}+u_{1}^{2}-\frac{3}{b} u_{1} \sin ^{2} \phi\right] .
\end{aligned}
$$

Noting that the integration constants of Eqs. (5) and (6) are chosen such that maximizing $u$ (or minimizing $r$ ) for $\phi=\pi / 2$, then we obtain the trajectory of photon up to the second-order in $G$ as,

$$
\begin{aligned}
\frac{1}{r}= & \frac{1}{b} \sin \phi+\frac{r_{g}}{4 b^{2}}(3+\cos 2 \phi) \\
& +\frac{r_{g}^{2}}{64 b^{3}}(37 \sin \phi+30(\pi-2 \phi) \cos \phi-3 \sin 3 \phi)
\end{aligned}
$$

where $b$ is the impact parameter which represents the minimum value of $r$-coordinate for the undeflected light ray, i.e., $r_{g}=0$. The bending angle $\alpha$ is shortly derived from Eq. (7) and it coincides with the famous formula by [13],

$$
\begin{aligned}
\alpha & =2 \frac{r_{g}}{b}+\frac{15 \pi}{16}\left(\frac{r_{g}}{b}\right)^{2} \\
& =\frac{4 G M}{c^{2} b}+\frac{15 \pi}{4} \frac{(G M)^{2}}{c^{4} b^{2}}+\mathcal{O}\left(G^{3}\right) .
\end{aligned}
$$

A simple derivation of $\alpha$ is given in Appendix B It should be mentioned about the validity of the solution for light trajectory. The appropriateness of our solution, Eq. (7) can be verified readily by the direct substitution into Eq. (2) and it is found that the residual terms are order $\mathcal{O}\left(G^{3}\right)$, then it is perfectly valid up to the order of $G^{2}$. However, the photon trajectory given in previous works such as Eq. (18) of [14] and Eq. (16) of [5] are incorrect; in fact, there appears $\mathcal{O}\left(G^{2}\right)$ order residual term in the solution of photon trajectory in [5, 14].

\section{CONTRIBUTION OF THE COSMOLOGICAL CONSTANT}

Now, let us investigate the contribution of $\Lambda$ on light ray. For this purpose, we adopt the Schwarzschild-de Sitter or Kottler metric [15],

$$
\begin{aligned}
d s^{2}= & -\left(1-\frac{r_{g}}{r}-\frac{\Lambda}{3} r^{2}\right) c^{2} d t^{2} \\
& +\left(1-\frac{r_{g}}{r}-\frac{\Lambda}{3} r^{2}\right)^{-1} d r^{2}+r^{2}\left(d \theta^{2}+\sin ^{2} \theta d \phi^{2}\right)
\end{aligned}
$$

It is clear that the trajectory of photon Eq. (12) strictly satisfies Eq. (10) up to the second-order in $G$ based on

the result in previous section.

In the same way as the Schwarzschild case, the differential equation of light is given by

$$
\left(\frac{d u}{d \phi}\right)^{2}=\frac{1}{b^{2}}-u^{2}+r_{g} u^{3}+\frac{\Lambda}{3} .
$$

It should be emphasized here that the geodesic equation of light Eq. (10) does obviously include $\Lambda$. Therefore, previous arguments, such as " $\Lambda$ does not appear in the geodesic equation of light", would be overstated. Actually, the second-order ODE derived from Eq. (10) reduces to Eq. (3), nevertheless its solution of light trajectory should be obtained in such a way that the integration constants satisfy Eq. (10).

Furthermore, the impact parameter is the minimum value of the coordinate $r$ if the light ray were undeflected, i.e., $r_{g}=0$. It is obvious from Eq. (10) that the impact parameter $B$ in this case is defined by

$$
\frac{1}{B^{2}} \equiv \frac{1}{b^{2}}+\frac{\Lambda}{3} \text {. }
$$

Then, the form of Eq. (10) completely coincides with Eq. (2), except that the impact parameter $b$ is replaced by $B$. Therefore, the solution of Eq. (10) becomes,

$$
\begin{aligned}
\frac{1}{r}= & \frac{1}{B} \sin \phi+\frac{r_{g}}{4 B^{2}}(3+\cos 2 \phi) \\
& +\frac{r_{g}^{2}}{64 B^{3}}(37 \sin \phi+30(\pi-2 \phi) \cos \phi-3 \sin 3 \phi)
\end{aligned}
$$

and deflection angle is,

$$
\begin{aligned}
\alpha & =2 \frac{r_{g}}{B}+\frac{15 \pi}{16}\left(\frac{r_{g}}{B}\right)^{2} \\
& =\frac{4 G M}{c^{2} B}+\frac{15 \pi}{4} \frac{(G M)^{2}}{c^{4} B^{2}}+\mathcal{O}\left(G^{3}\right) .
\end{aligned}
$$

It is worthy to note that the contribution of $\Lambda$ is incorporated in Eqs. (12) and (13) through Eq. (11). As a consequence, it is found that the cosmological constant $\Lambda$ does appears in both the geodesic equation and its solution, that is the trajectory of photon. However, the effect of $\Lambda$ is completely absorbed into the definition of the the impact parameter (see Eq. (11)). Hence it is difficult to distinguish the influence of $\Lambda$ from the observed deflection angle.

When we expand Eq. (13) by using $1 / B=$ $(1 / b) \sqrt{1+\Lambda b^{2} / 3} \simeq(1 / b)\left(1+\Lambda b^{2} / 6\right)$ and remain $\mathcal{O}(M, M \Lambda)$ terms, it follows that

$$
\alpha \simeq \frac{4 G M}{c^{2} b}+\frac{2 G M b \Lambda}{3 c^{2}},
$$

in which second term coincides with the previous results, e.g. Eq. (5) and below in [16] and the third term of Eq. (15) in [10]. Hence it is found that these results are ncluded in Eq. (13) as a limiting case. 


\section{COSMOLOGICAL LENS EQUATION}

Finally, we consider the contribution of $\Lambda$ in the cosmological lens equation. Under the assumption that the thin lens approximation is valid, the lens equation relates the observed image position angle $\theta$ to the unlensed position angle $\beta$ of the source as

$$
\beta=\theta-\frac{D_{A}\left(z_{L}, z_{S}\right)}{D_{A}\left(0, z_{S}\right)} \alpha,
$$

where $D_{A}\left(z_{1}, z_{2}\right)$ denotes the angular diameter distance from the redshift $z_{1}$ to $z_{2}$, and the arguments $z_{L}$ and $z_{S}$ are the redshift of the lens and the source, respectively. For the distance formula $D_{A}$ in the unperturbed Friedmann-Lemaître-Robertson-Walker (FLRW) universe with $\Lambda$, see, e.g., [17].

Up to the first order in $G$, the bending angle $\alpha$ in the present case is

$$
\alpha=\frac{4 G M}{c^{2} B} .
$$

The impact parameter $B$ is related to the image position angle $\theta$ by

$$
B=D_{A}\left(0, z_{L}\right) \theta .
$$

Then, from Eqs. (15), (16), and (17), the lens equation is finally

$$
\beta=\theta-\frac{4 G M D_{A}\left(z_{L}, z_{S}\right)}{c^{2} D_{A}\left(0, z_{L}\right) D_{A}\left(0, z_{S}\right)} \frac{1}{\theta} .
$$

Therefore, the contribution of $\Lambda$ is completely involved in the form of the angular diameter distance $D_{A}$. No modifications due to $\Lambda$ appear even if the term of $\mathcal{O}\left(G^{2}\right)$ in $\alpha$ is included. It should also be noted that Eq. (18) is exactly the same form appeared in [17, 18], where the authors have shown that the gravitational lensing effects are strongly dependent on the value of the cosmological constant and hence they provide us with useful means to test the cosmological constant.

Here, we mention that in paper [16], Sereno introduced the relation, $b_{0}=D_{d} \vartheta$ (see Eq. (8) below of [16]) where $b_{0}$ is the impact factor of "Schwarzschild lens" (see Eq. (8) of [16]), $D_{d}$ is the angular-diameter distance, and $\vartheta$ is the angular separation. Since in the case of cosmological lens, the cosmological distance, such as the angular diameter distance, is defined with $\Lambda$, then $b_{0}$ should be replaced by $B$, instead.

\section{SUMMARY}

We briefly summarize our conclusions. Contrary to the previous arguments, (a) the cosmological constant $\Lambda$ does appear in the orbital equation of light, (b) nevertheless the bending angle of light $\alpha$ does not change its form even if $\Lambda \neq 0$ since the contribution of $\Lambda$ is thoroughly absorbed into the definition of the impact parameter, and (c) the effect of $\Lambda$ is completely involved in the angular diameter distance $D_{A}$. Then, no modifications due to $\Lambda$ appear even if the second-order terms in $G$ are included.

It should be mentioned that the similar conclusions are reached by other authors [6 [9]. We hope that this paper provides a simpler and clearer explanation of the role of $\Lambda$ in the bending of light and the cosmological lens equation than the above-mentioned studies.

\section{Appendix A: The angle between the radial direction and the light trajectory}

Rindler and Ishak [4] have derived the angle between the radial direction and the light trajectory from the invariant cosine formula. Just for a reference, here we give a more intuitive derivation. Consider a spherically symmetric space. The metric on the equatorial plane $\theta=\pi / 2$ is given by

$$
d \ell^{2}=\frac{d r^{2}}{f(r)}+r^{2} d \phi^{2}
$$

In this space, the infinitesimal proper lengths along the radial direction and that perpendicular to the radial direction are given by $f(r)^{-1 / 2}|d r|$ and $r|d \phi|$, respectively. Then the tangent of the angle $\psi$ between the radial direction and the light trajectory is

$$
\tan \psi=\frac{r|d \phi|}{f(r)^{-1 / 2}|d r|}=\sqrt{f(r)} r\left|\frac{d r}{d \phi}\right|^{-1},
$$

which is the same form as Eq. (16) in [4]. Once we obtain the solution $r(\phi)$ for the light trajectory, Eq. (A2) gives the angle at any point $(r(\phi), \phi))$ on the trajectory.

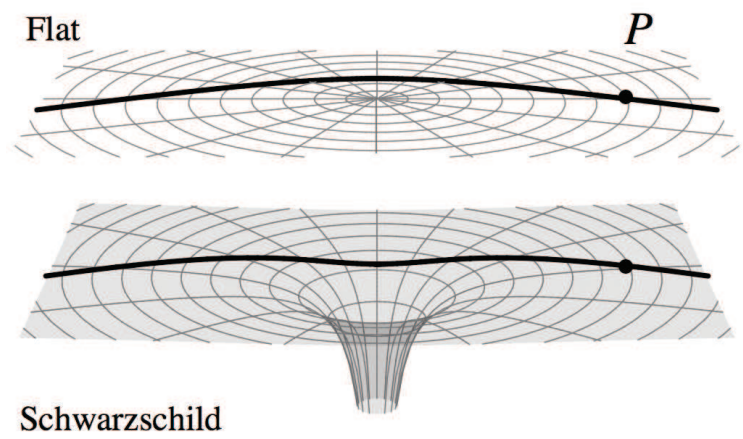

FIG. 1: Light trajectories on Schwarzschild geometry and on the flat space which is tangent to the asymptotically flat region of the Schwarzschild geometry. The coordinates of the point $P$ is $(r(\phi), \phi))=(r(0), 0)$. 


\section{Appendix B: The bending angle in Schwarzschild geometry}

Using Eqs. (11), (7) and (A2), the angle $\psi$ at the point $P(r(0), 0)$ is

$$
\psi \simeq \sqrt{f(r(0))} r(0)\left|\frac{d r}{d \phi}\right|_{\phi=0}^{-1} \simeq \frac{r_{g}}{b}+\frac{15 \pi}{32}\left(\frac{r_{g}}{b}\right)^{2} .
$$

Consider a flat space which is tangent to the asymptotically flat region of the Schwarzschild geometry (see Fig. 11). It is apparent from the property of an isosceles triangle in the Euclidean geometry that the angle $\psi$ at the point $P$ is a half of the bending angle $\alpha$ (see Fig. 21). Then,

$$
\alpha=2 \psi \simeq 2 \frac{r_{g}}{b}+\frac{15 \pi}{16}\left(\frac{r_{g}}{b}\right)^{2} .
$$

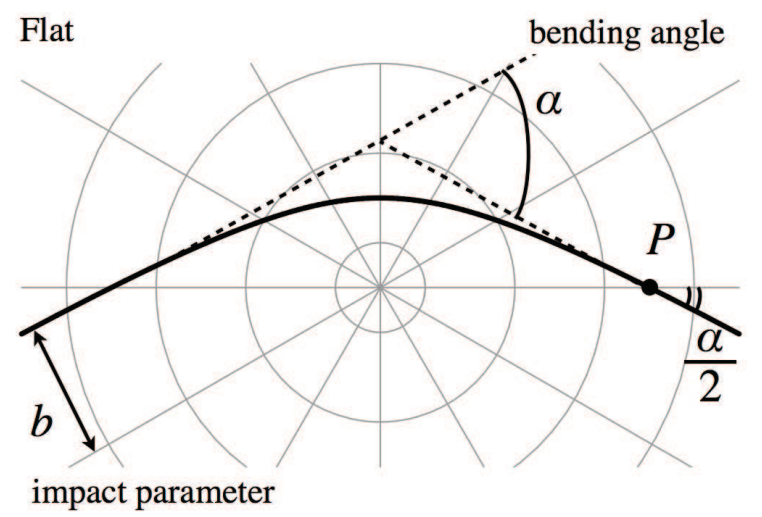

FIG. 2: Light trajectory on the flat space with the metric $d \ell^{2}=d r^{2}+r^{2} d \phi^{2}$. The impact parameter $b$ and the bending angle $\alpha$ are defined on this space.

\section{Flat}
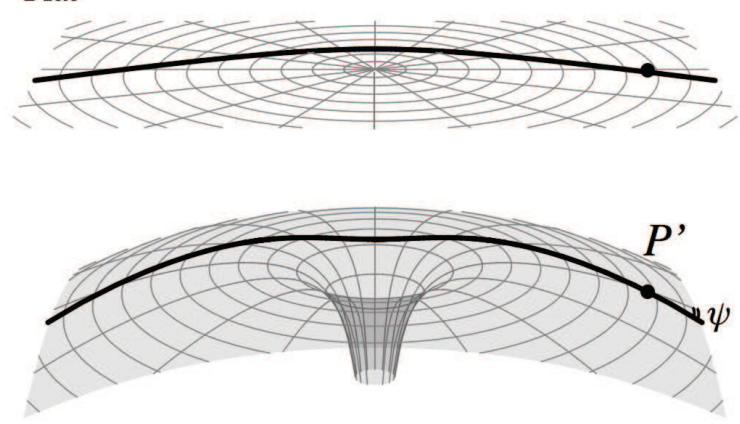

\section{Schwarzschild-de Sitter/Kottler}

FIG. 3: The angle $\psi$ between the radial direction and the light trajectory at $P^{\prime}$ on Schwarzschild-de Sitter geometry.

\section{Appendix C: The angle on Schwarzschild-de Sitter geometry}

Using Eqs. (91), (12), and (A2), the angle $\psi$ between the radial direction and the light trajectory at the point $P^{\prime}(r(0), 0)$ on the Schwarzschild-de Sitter geometry is (see Fig. 3)

$$
\psi \simeq \sqrt{1-\frac{\Lambda}{3}\left(\frac{b^{2}}{r_{g}}\right)^{2}}\left(\frac{r_{g}}{b}+\frac{15 \pi}{32}\left(\frac{r_{g}}{b}\right)^{2}\right) .
$$

When linearized with respect to $\Lambda$,

$$
\psi \simeq \frac{r_{g}}{b}+\frac{15 \pi}{32}\left(\frac{r_{g}}{b}\right)^{2}-\frac{\Lambda}{6} \frac{b^{3}}{r_{g}}-\frac{15 \pi}{192} \Lambda b^{2},
$$

which is essentially a half of Eq. (18) in [5], although they drop the last term in Eq. (C2). It should be emphasized since the Euclidean property does not hold on the Schwarzschild-de Sitter geometry, the angle $\psi$ in Eq. (C2) is not a half of the bending angle $\alpha$. 
[1] A. W. Kerr, J. C. Hauck, B. Mashhoon, Class. Quant. Grav., 20, 2727 (2003).

[2] W. Rindler, Relativity: Special, General, and Cosmological (2nd Ed.), Oxford Univ. Press, New York (2006).

[3] L. Iorio, Adv. Astron., id. 268647 (2008).

[4] W. Rindler, M. Ishak, Phys. Rev. D, 76, id. 043006 (2007)

[5] M. Ishak, W. Rindler, Gen. Rel. Grav., 42, 2247 (2010).

[6] K. Lake, Phys. Rev. D. 65, id. 087301 (2002).

[7] M. Park, Phys. Rev. D, 78, id. 023014 (2008).

[8] I. B. Khriplovich, A. A. Pomeransky, Int. J. Mod. Phys. D, 17, 2255 (2008).

[9] F. Simpson, J. A. Peacock, A. F. Heavens, MNRAS, 402, 2009 (2010).

[10] A. Bhadra, S. Biswas, K. Sarkar, Phys. Rev. D, 82, id.
063003 (2010).

[11] H. Miraghaei, M. Nouri-Zonoz, Gen. Rel. Grav. 42, 2947 (2010)

[12] T. Biressa, J. A. de Freitas Pacheco, Gen. Rel. Grav., 43, 2649 (2011).

[13] R. Epstein, I. I. Shapiro, Phys. Rev. D, 22, 2947 (1980).

[14] J. Bodenner, C. M. Will, Am. J. Phys., 71, 770 (2003).

[15] F. Kottler, Annalen. Phys., 361, 401 (1918).

[16] M. Sereno, Phys. Rev. Lett., 102, id. 021301 (2009).

[17] M. Fukugita, T. Futamase, M. Kasai, E. L. Turner, Astrophys. J., 393, 3 (1992).

[18] M. Fukugita, T. Futamase, M. Kasai, Mon. Not. R. Astr. Soc., 246, 24 (1990). 